\title{
Streptococcus agalactiae infective endocarditis in Canada: a multicenter retrospective nested case control analysis
}

\author{
Torrance Oravec ${ }^{*}$, S. Annie Oravec ${ }^{2}$, Jennifer Leigh ${ }^{3}$, Liam Matthews ${ }^{4}$, Bahareh Ghadaki ${ }^{5}$, Dominik Mertz ${ }^{6}$, \\ Peter Daley ${ }^{7}$ and Anjali Shroff ${ }^{6}$
}

\begin{abstract}
Background: Infective endocarditis (IE) caused by Streptococcus agalactiae (GBS) is increasingly reported and associated with an aggressive course and high mortality rate. Existing literature on GBS IE is limited to case series; we compared the characteristics of patients with GBS IE to patients with GBS bacteremia without IE to identify risk factors for development of IE.
\end{abstract}

Methods: A nested case-control study in a cohort of adult patients with GBS bacteremia over a 18-year period was conducted across seven centres in three Canadian cities. A chart review identified patients with possible or definite IE (per Modified Duke Criteria) and patients with IE were matched to those without endocarditis in a 1:3 fashion. Multivariate analyses were completed using logistic regression.

Results: Of 520 patients with GBS bacteremia, 28 cases of possible or definite IE were identified (5.4\%). 68\% (19/28) met criteria for definite IE, surgery was performed in 29\% (8/28), and the overall in-hospital mortality rate was 29\% (8/28). Multivariate analysis demonstrated that IE was associated with injection drug use $(\mathrm{OR}=19.6,95 \% \mathrm{Cl}=3.39$ $111.11, p=0.001)$, prosthetic valve $(\mathrm{OR}=11.5,95 \% \mathrm{Cl}=1.73-76.92, \mathrm{p}=0.011)$ and lack of identified source of bacteremia $(\mathrm{OR}=3.81,95 \% \mathrm{Cl}=1.24-11.65, \mathrm{p}=0.019)$.

Conclusions: GBS bacteremia, especially amongst people who inject drugs, those with prosthetic valves, and those with no apparent source of infection, should increase clinical suspicion for IE.

Keywords: Streptococcus agalactiae, Group B Streptococcus, Infective endocarditis, Case control, Retrospective

\section{Background}

Serious systemic infections due to Streptococcus agalactiae (Group B Streptococcus-GBS) in non-pregnant adults are increasingly reported [1-4]; a recent multinational population-based assessment demonstrated increased incidence for invasive GBS infection, driven by an increase in adults over 60 [5]. GBS is known to

\footnotetext{
*Correspondence: torrance.oravec@medportal.ca

${ }^{1}$ Division of Infectious Diseases, Department of Medicine, University of British Columbia, 328C Heather Pavilion E, 2733 Heather St. Vancouver, Vancouver, BC V5Z 3J5, Canada

Full list of author information is available at the end of the article
}

cause invasive disease in pregnancy, the peripartum period, and in neonates [6], but other disease manifestations include pneumonia, skin and soft tissue infection, osteomyelitis, joint infection, abscess, meningitis, endocarditis and bacteremia without focus $[2,3]$. Numerous risk factors for the development of invasive GBS disease in non-pregnant adults have been identified, particularly diabetes mellitus, but also other immunocompromising states, active malignancy, and advanced age [2, 3, 7-9].

GBS has been increasingly been reported as a cause of Infective endocarditis (IE) and is of particular interest because of its association with an aggressive course, 
highly destructive effect on valvular tissue, and high mortality rate $[5,10-13]$. Description of GBS IE is limited to case series which have examined the epidemiology, natural history, and complications of this disease, and provide insight into how the disease has been treated in specific circumstances $[4,10,13,14]$. More recent analyses have demonstrated that GBS is associated with an aggressive IE phenotype in comparison to other Streptococcus species, and is likely the most common beta-hemolytic Streptococcus causing IE [12]. No studies have examined risk factors for the development of IE amongst individuals with invasive GBS disease.

We present a retrospective, nested case-control analysis of GBS IE and GBS bacteremia, to compare these groups and to describe risk factors for the development of IE.

\section{Methods}

\section{Patient population}

Our study was conducted across seven hospitals in three Eastern Canadian cities (Hamilton and Niagara, both in Ontario, and St John's in Newfoundland). Patient records from January 1, 2000 to December 31, 2018 were reviewed. Ethics approval was obtained from local Research Ethics Boards with waiver of patient consent.

All patients of at least 18 years of age that had blood cultures positive for Streptococcus agalactiae were identified through local microbiology labs, and medical records were screened for the diagnosis of IE. Cases were identified if they met the Modified Duke Criteria (chosen given widespread use in previous literature) for possible or definite IE, and controls either did not meet criteria or had the diagnosis rejected [15]; some cases were rejected despite meeting Modified Duke Criteria due to presence of a firm alternate diagnosis or resolution of symptoms within four days. For each case we identified three controls, matched by study site and proximity of bacteremia in time to the corresponding case. It was felt that matching cases to controls who presented to the same hospital at a similar time would help to control for resource availability and management strategies.

\section{Data collection and outcomes}

Data was collected using a standardized case report form and de-identified prior to analysis. Risk factors for GBS IE identified in previous case series were included, as were generally accepted risk factors for IE and for invasive GBS infections [10,12, 15]. Outcomes were compared including mortality, congestive heart failure, cardiac conduction abnormalities, neurological complications, and need for valvular surgery.

\section{Statistical analysis}

We compared cases and controls using a paired t-test for continuous variables and Pearson's test for categorical variables. We used Cox-Snell binary logistic regression (conditional, forward step-wise) for multivariate analysis, including all variables with $\mathrm{p}<0.05$ from univariate analysis. Statistical analysis was performed on SPSS version 25 (IBM, USA). Cases with missing data were not included in risk factor analysis, nor were matched controls.

\section{Results}

Five hundred twenty patients with a total of 827 positive blood cultures were included. $28 / 520$ patients $(5.4 \%)$ met the case definition of IE (19 definite IE, 9 possible IE). A single case was identified during the years 2000-2010, with the remaining 27 cases identified from 2011 to 2018 . 84 matched controls were identified.

Demographic and baseline clinical characteristics of cases and controls are presented in Table 1. Cases and controls were similar in terms of age, sex and comorbidities. Cases were significantly more likely to have valvular disease at baseline than controls (35.7 vs $8.3 \%$; $\mathrm{p}<0.001)$, more likely to have a prosthetic valve (17.9 vs $2.4 \% ; \mathrm{p}=0.003)$, more likely to have a history of injection drug use ( 25.0 vs. $2.4 \% ; \mathrm{p}<0.001)$, and more likely to have a history of alcohol use disorder (14.3 vs $1.2 \%$; $=0.004$ ).

The clinical course and outcome of cases and controls are detailed in Table 2. Invasive GBS tended to present acutely in both cases and controls, with average days of symptoms before presentation of 4.9 and 4.2, respectively $(p=0.64)$. Cases did not have significantly shorter time to culture positivity ( 11.8 vs $15.5 \mathrm{~h} ; \mathrm{p}=0.17$ ), but had a greater number of positive blood culture sets $(2.9$ vs. $1.7 ; \mathrm{p}<0.001)$ as they had more blood culture sets drawn ( 2.5 vs $2.1 ; \mathrm{p}=0.048)$. All cases were community acquired, whereas five controls had nosocomial infections $(\mathrm{p}=0.19)$. Cases were not significantly more likely to have had a recent procedure (10.7 vs $3.6 \% ; \mathrm{p}=0.15)$. No significant differences were observed in rates of concurrent skin, soft tissue, bone or joint infection, nor rates of indwelling lines. Amongst controls, skin and soft tissue infection was the source in 33 patients, respiratory infection in 9, genitourinary in 13 , osteoarticular in 8 , and central line associated in 1;20 controls did not have a source identified.

Cases were more likely to have an echocardiogram done than controls $(85.7 \%$ vs $45.2 \% ; \mathrm{P}<0.001)$. Ten cases also received transesophageal echocardiography, whereas only four controls did. Four cases did not have an echocardiogram but met Modified Duke's Criteria for "possible endocarditis); two of these cases died early in the treatment course, and the other two received relatively short courses of therapy. Cases were 
Table 1 Demographic and baseline clinical characteristics of Group B Streptococcus infective endocarditis cases and matched controls

\begin{tabular}{llll}
\hline & $\begin{array}{l}\text { Cases } \\
(\mathbf{N}=\mathbf{2 8})\end{array}$ & $\begin{array}{l}\text { Controls } \\
\mathbf{( N = 8 4 )}\end{array}$ & $\begin{array}{l}\text { (Pearson 2-sided } \\
\text { Chi-square, or 2-tailed } \\
\text { t test) }\end{array}$ \\
\hline Age in years (mean \pm SD) & & $\mathrm{P}=0.066$ \\
Females & $59.0 \pm 16.8$ & $65.4 \pm 15.5$ & $\mathrm{P}=0.58$ \\
Non-valvular cardiac disease & $14(50.0 \%)$ & $37(44.0 \%)$ & $\mathrm{P}=0.84$ \\
Atrial fibrillation & $11(39.3 \%)$ & $34(40.5 \%)$ & $\mathrm{P}=0.49$ \\
Coronary artery disease & $4(14.3 \%)$ & $17(20.2 \%)$ & $\mathrm{P}=0.46$ \\
Congestive heart failure & $9(32.1 \%)$ & $21(25.0 \%)$ & $\mathrm{P}=0.70$ \\
Valvular disease & $2(7.1 \%)$ & $8(9.5 \%)$ & $\mathrm{P}<0.001$ \\
Cardiac device & $10(35.7 \%)$ & $7(8.3 \%)$ & $\mathrm{P}=0.24$ \\
Prosthetic valve & 0 & $4(4.8 \%)$ & $\mathrm{P}=0.003$ \\
Diabetes & $5(17.9 \%)$ & $2(2.4 \%)$ & $\mathrm{P}=0.48$ \\
Active malignancy & $12(42.9 \%)$ & $43(51.2 \%)$ & $\mathrm{P}=0.86$ \\
Immunocompromised & $3(10.7 \%)$ & $8(9.5 \%)$ & $\mathrm{P}=0.68$ \\
Pregnant & $3(10.7 \%)$ & $12(14.3 \%)$ & $\mathrm{P}=0.41$ \\
Post-partum & $1(3.6 \%)$ & $1(1.2 \%)$ & $\mathrm{P}=0.31$ \\
Cirrhosis & 0 & $3(3.6 \%)$ & $\mathrm{P}=0.55$ \\
Genitourinary disease & $3(10.7 \%)$ & $6(7.1 \%)$ & $\mathrm{P}=0.57$ \\
Chronic kidney disease & $4(14.3 \%)$ & $16(19.0 \%)$ & $\mathrm{P}=0.78$ \\
Hemodialysis & $6(21.4 \%)$ & $16(19.0 \%)$ & $\mathrm{P}=0.63$ \\
Injection drug use & $1(3.6 \%)$ & $5(6.0 \%)$ & $\mathrm{P}<0.001$ \\
Alcohol use disorder & $7(25.0 \%)$ & $2(2.4 \%)$ & $\mathrm{P}=0.004$ \\
\hline
\end{tabular}

*HIV/AIDS, neutropenia, organ transplantation, immunoglobulin deficiency, immunosuppressive therapy (chemotherapy, radiotherapy, anti-TNF-alpha, DMARDs, or immunosuppressive biologics), splenectomy/asplenia

also more likely to receive consultation from an Infectious Disease specialist (75\% vs $32.1 \%$; $<0.001$ ). Cases were more likely to have recurrent bacteremia, defined as blood cultures positive for GBS following documented negative cultures ( $14.3 \%$ vs $0 \% ; \mathrm{p}<0.001)$, and were less likely to have a source identified ( $50 \%$ vs $77 \%$; $\mathrm{p}=0.006$ ). Patients with GBS IE received 45.1 days of antibiotics on average, while patients without endocarditis received an average of 19.9 days of antibiotics $(\mathrm{p}<0.001)$.

Description of cases is presented in Table 3. Of cases that had echocardiograms performed, $50 \%$ had aortic valve infection, with tricuspid valve infection being second most common (33.3\%) followed by mitral valve (20.8\%). No patients had documented pulmonic valve involvement, and a single patient had both mitral and tricuspid endocarditis. High rates of complications were observed among cases: $32.1 \%$ had acute heart failure, $32.1 \%$ had neurologic complications (embolic stroke or epidural abscess), $16.7 \%$ had valve perforation, $12.5 \%$ had an intracardiac abscess, and $10.7 \%$ had cardiac conduction system disease. In-hospital mortality was significantly higher in endocarditis cases than in controls $(28.6 \%$ vs $3.6 \%$; $p<0.001)$. Surgery was performed in $28.6 \%$ of cases, and all patients undergoing surgery survived to discharge.

\section{Multivariate analysis}

Results of multivariate analysis are presented in Table 4. One case and three controls were excluded from multivariate analysis because of missing data. Regression proceeded over four cycles, at which point injection drug use (OR for $\mathrm{IE}=19.6,95 \% \mathrm{CI}=3.39$ $111.11, \mathrm{p}=0.001)$, prosthetic valve (OR for $\mathrm{IE}=11.5$, 95\% $\mathrm{CI}=1.73-76.92, \mathrm{p}=0.011)$ and lack of identified source of bacteremia (OR for $\mathrm{IE}=3.81,95 \% \mathrm{CI}=1.24$ $11.65, \mathrm{p}=0.019)$ emerged as significant predictors for the development of IE.

1 case and 3 controls excluded for missing data

Binary logistic regression (conditional, forward step-wise) included: native valve disease (categorical), injection drug use (categorical), alcohol use disorder (categorical), recent procedure (categorical), prosthetic valve (categorical), time to positivity (continuous), community acquired (categorical), recurrent bacteremia (categorical), source identified (categorical). 
Table 2 Clinical course and outcomes for GBS IE cases and controls

\begin{tabular}{|c|c|c|c|}
\hline & $\begin{array}{l}\text { Cases } \\
(\mathrm{N}=28)\end{array}$ & $\begin{array}{l}\text { Controls } \\
(\mathrm{N}=84)\end{array}$ & $\begin{array}{l}\text { (Pearson } \\
\text { 2-sided } \\
\text { Chi-square, } \\
\text { or 2-tailed t } \\
\text { test) }\end{array}$ \\
\hline $\begin{array}{l}\text { Mean days of symptoms } \\
\text { Before diagnosis }\end{array}$ & $4.9 \pm 5.9$ & $4.2 \pm 7.2$ & $P=0.64$ \\
\hline $\begin{array}{l}\text { Mean time to culture } \\
\text { Positivity (hours) }\end{array}$ & $11.8 \pm 6.2$ & $15.5 \pm 13.2$ & $P=0.17$ \\
\hline $\begin{array}{l}\text { Mean number of positive } \\
\text { Blood culture sets }\end{array}$ & $2.9 \pm 2.6$ & $1.7 \pm 0.7$ & $P<0.001$ \\
\hline $\begin{array}{l}\text { Mean number of blood } \\
\text { Culture sets collected }\end{array}$ & $2.5 \pm 0.5$ & $2.1 \pm 0.4$ & $P=0.048$ \\
\hline Community acquired & $28(100 \%)$ & 79 (94.0\%) & $P=0.19$ \\
\hline Concurrent skin or soft tissue infection & $8(28.6 \%)$ & $34(40.5 \%)$ & $P=0.26$ \\
\hline Concurrent bone or joint infection & $6(21.4 \%)$ & $15(17.9 \%)$ & $P=0.68$ \\
\hline Recurrent bacteremia & $4(14.3 \%)$ & 0 & $P<0.001$ \\
\hline Source identified & $14(50.0 \%)$ & $65(77.0 \%)$ & $P=0.006$ \\
\hline Recent procedure* & $3(10.7 \%)$ & $3(3.6 \%)$ & $P=0.15$ \\
\hline Indwelling line & $1(3.6 \%)$ & $3(3.6 \%)$ & $P=0.96$ \\
\hline Echo done & $24(85.7 \%)$ & 38 (45.2\%) & $P<0.001$ \\
\hline ID consult & $21(75.0 \%)$ & $27(32.1 \%)$ & $P<0.001$ \\
\hline \multicolumn{4}{|l|}{ Outcomes } \\
\hline In hospital mortality & $8(28.6 \%)$ & $3(3.6 \%)$ & $P<0.001$ \\
\hline Mean duration of antibiotics (days) & $45.1 \pm 40.4$ & $19.9 \pm 15.7$ & $P<0.001$ \\
\hline
\end{tabular}

*Any surgeries, invasive procedures in past 3 months, including PICC/central line insertion, endoscopy, suprapubic catheter insertion, C-section, therapeutic abortion

Table 3 Clinical characteristics and complications occurring in GBS IE cases

\begin{tabular}{lc}
\hline & $\begin{array}{l}\text { Cases } \\
(\mathbf{N}=\mathbf{2 8})\end{array}$ \\
\hline Aortic valve & $12(50 \%)^{*}$ \\
Mitral valve & $5(20.8 \%)^{*}$ \\
Tricuspid valve & $8(33.3 \%)^{*}$ \\
Valve perforation & $4(16.7 \%)^{*}$ \\
Intracardiac abscess & $3(12.5 \%)^{*}$ \\
Congestive heart failure & $9(32.1 \%)$ \\
Conduction disease & $3(10.7 \%)$ \\
Neurological complication & $9(32.1 \%)$ \\
Surgery & $8(28.6 \%)$ \\
\hline
\end{tabular}

*Percentages for valves involved and valvular complications use a denominator of 24, as no echo was done for 4 patients (IE diagnosed without echocardiographic criteria). A single case had both mitral and tricuspid valve involvement

Four iterations.

Step $4 \mathrm{R}^{2}=0.305$ (Cox and Snell).
Table 4 Multivariate analysis of risk factors for the development of IE amongst patients with GBS bacteremia

\begin{tabular}{llll}
\hline Risk factor & OR & $\mathbf{9 5 \% ~ C l}$ & p value \\
\hline Injection drug use & 19.6 & $3.39-111.11$ & 0.001 \\
Prosthetic valve & 11.5 & $1.73-76.92$ & 0.011 \\
Recurrent bacteremia & 0 & N/A & 0.99 \\
Source identified & 0.26 & $0.085-0.80$ & 0.019 \\
\hline
\end{tabular}

Excluded from model $(\mathrm{p}>0.05)$ : native valve disease (categorical), alcohol use disorder (categorical), recent procedure (categorical), time to positivity (continuous), community acquired (categorical).

Model predicts $95.1 \%$ of controls and $55.6 \%$ of cases.

\section{Discussion}

Our retrospective nested case control study found that injection drug use, presence of a prosthetic valve, and lack of apparent source were all risk factors for IE among patients with GBS bacteremia, and provides a novel perspective on the clinical characteristics of GBS IE. Mortality amongst cases was significantly higher than in controls, and rates of systemic complications 
were similarly high. Interestingly, many previously identified risk factors for the development of invasive GBS disease (diabetes, immunocompromise, malignancy, advanced age) were not significant predictors of IE, illustrating the distinct pathophysiology of endovascular infections $[3,8,9]$.

Amongst our study population, $5.4 \%$ of patients with Streptococcus agalactiae blood stream infections developed IE, which is roughly consistent with previously reported incidence of IE amongst GBS bacteremia (8.5\%) [13] and amongst invasive GBS infections overall (3.0$10.5 \%)[1,3]$. During the eighteen years of our study, the annual incidence of GBS IE increased, with $27 / 28$ cases $(96.4 \%)$ occurring in the later seven years, a trend which has been observed at other centres $[2,3,13,14]$. The overall incidence of invasive GBS disease has also increased across populations in Australia, Canada, Denmark, Sweden, Finland and the UK $[5,6,16]$. Population level surveillance explains this increase based on aging [5], but higher rates of comorbidities (diabetes, immunosuppression and malignancy) $[8,9]$, reduced physical capacity, and altered host immune response may also contribute [7].

Our GBS IE cases demonstrated a left side predominance, which has been reported consistently across multiple studies $[4,10,12,13]$. We observed high rates of complications, similar to previous reports [12]. The tendency for GBS to cause systemic embolism has been attributed to the tendency towards large, friable vegetations, which have themselves been related to the capacity for GBS to bind fibrinogen and platelets [17]. A similar mechanism of virulence has been proposed for the pathogenesis of the less common entity of Streptococcus pyogenes IE [18].

We observed an in-hospital mortality rate among cases of $28.6 \%$. Mortality was $85 \%$ in the pre-antibiotic era [4], and mortality reported in case series from the 1960's to the 1990 's ranged from 40.7 to $85.7 \%$, though many of these series involved less than 10 patients $[4,10,11,13$, $14,19]$. Sambola et al. conducted a review of 115 cases of GBS IE and described mortality as 45\% between 1962 and 1979, and 34\% between 1980 and 1998. In studies that report data collected after the year 2000, mortality has ranged from 20 to $33.3 \%$, with the relative reduction in mortality attributed to increased recognition, improved diagnosis, and early surgical intervention $[12,20]$. The rate of surgical intervention previously reported for GBS IE is highly variable (14.3-83.3\%), and the relatively low mortality rate in our study is not clearly related to differences in rates of surgical intervention [4, 20]. The lower mortality rate in our study may be related to an increased proportion of young patients with intravenous drug use as a risk factor (as opposed to older, comorbid diabetic patients described in other studies) [10].

The most novel aspect of our study is that the nested case-control design allows assessment of factors which may predispose individuals who have GBS bacteremia towards developing IE, a type of analysis that has not previously been reported. Based on our multivariate analysis, independent predictors for the development of IE include injection drug use, having a prosthetic valve, and not having a clearly identified source of bacteremia. This is not particularly surprising, as injection drug use and prosthetic valves are so well recognized as conditions predisposing to IE that they are included in the Modified Duke Criteria [15]. Of note, a greater proportion of people who inject drugs were present in our study than have been reported in most other GBS IE case series (two of the seven patients described by Gallagher et al., otherwise rates have been 3.2-8.3\%) [4, 12-14]. While local variations in demographic factors may contribute, our data are the most contemporary available, and our relatively high proportion of people who inject drugs is likely due, at least in part, to the ongoing opiate epidemic in Canada [21].

Conditions which have been previously associated with invasive GBS (diabetes, increased age, active malignancy and immunosuppression) did not contribute to risk of developing IE in our study $[5,7,8]$. This may be due to the fact that these conditions predispose to GBS bacteremia but do not impact risk of IE.

Limitations of our design include retrospective data collection, which may have misidentified cases and controls based on missing data in the medical records. While our study does have a relatively high number of cases compared to previous reports, our case numbers were still limited; larger numbers would allow for more robust conclusions to be drawn. Our limited case numbers also prevented determination of differences in outcome due to antimicrobial choice. Our study was conducted exclusively in Canada, and generalizability to other regions is not assured. Not all cases and controls received an echocardiogram, so additional cases may have been identified if every patient underwent the same investigations, though we did not observe any recurrence of bacteremia in controls who had previously been admitted for GBS bacteremia without IE. Additionally, our use of Modified Duke Criteria to define cases may not reflect pragmatic treatment decisions; two of our cases did not receive echocardiograms, and were treated with short courses of therapy, indicating clinicians are not strictly following these criteria when making treatment decisions. Similarly, ID consultation was more likely in cases, and may have led to a more thorough diagnostic evaluation leading to a diagnosis of IE. 


\section{Conclusions}

Streptococcus agalactiae is an increasingly common cause of infective endocarditis and, coupled with a high mortality rate and markedly destructive phenotype, warrants consideration as a cause of IE. Presence of GBS bacteremia, especially amongst people who inject drugs, those with pre-existing valvular disease, and those with no apparent source of infection, should prompt clinicians to have a high suspicion for IE.

\section{Abbreviations}

DMARD: Disease modifying anti-rheumatic drugs; GBS: Group B Streptococcus, Streptococcus agalactiae; HIV/AIDS: Human Immunodeficiency Virus/Acquired Immunodeficiency Syndrome; ID: Infectious diseases; IE: Infective endocarditis.

\section{Acknowledgements}

We thank Raheel Inayatullah for conducting initial literature review.

\section{Authors' contributions}

PD, BG and AS served as local site leads. JL, LM, SO and TO conducted chart reviews at local institutions. PD and DM provided statistical guidance, and PD conducted statistical analysis. TO wrote the manuscript, with PD, DM and AS providing editorial support. All authors read and approved the final manuscript.

\section{Funding}

This study was conducted without funding. Article processing fees were partly funded by research grants awarded to PD, otherwise authors contributed independently.

\section{Availability of data and materials}

The datasets used and/or analysed during the current study are available from the corresponding author on reasonable request.

\section{Declarations}

\section{Ethics approval and consent to participate}

Ethics approval for retrospective chart review and data abstraction was obtained by authors from Research Ethics Boards at each study site: the Hamilton Integrated Research Ethics Board (McMaster University) granted approval in Hamilton (3734) and in Niagara (18-047-C); the Newfoundland and Labrador Health Research Ethics Board non-clinical trial subcommittee (Memorial University) granted approval in St. John's (2018.167). Need for patient consent was waived by each Research Ethics Board (given retrospective low risk nature of study). Data was irreversibly deidentified before being pooled for analysis. All research was performed in accordance with guidelines and regulations as laid out by respective research boards, and follows standard protocol for retrospective chart reviews with no patient contact and minimal risk.

\section{Consent for publication}

Not applicable.

\section{Competing interests}

The authors declare that they have no competing interests.

\section{Author details}

${ }^{1}$ Division of Infectious Diseases, Department of Medicine, University of British Columbia, 328C Heather Pavilion E, 2733 Heather St. Vancouver, Vancouver, BC V5Z 3J5, Canada. ${ }^{2}$ Division of Respirology, Department of Medicine, University of British Columbia, Vancouver, BC, Canada. ${ }^{3}$ Division of Internal Medicine, Department of Medicine, University of Ottawa, Ottawa, ON, Canada. ${ }^{4}$ Division of Internal Medicine, Department of Medicine, Memorial University of Newfoundland, St. John's, NL, Canada. 'Division of Infectious Diseases, Department of Medicine, Halton Healthcare, Oakville, ON, Canada. ${ }^{6}$ Division of Infectious Diseases, Department of Medicine, McMaster University, Hamilton, ON
Canada. ${ }^{7}$ Division of Infectious Diseases, Department of Medicine, Memorial University of Newfoundland, St. John's, NL, Canada.

Received: 8 September 2021 Accepted: 15 December 2021

Published online: 04 January 2022

\section{References}

1. Tazi A, Morand PC, Réglier-Poupet H, Dmytruk N, Billoët A, Antona D, et al. Invasive group B streptococcal infections in adults, France (2007-2010). Clin Microbiol Infect. 2011. https://doi.org/10.1111/j.1469-0691.2011. 03628.x.

2. Farley MM. Group B streptococcal disease in nonpregnant adults. Clin Infect Dis. 2001. https://doi.org/10.1111/j.1469-0691.2011.03628.x.

3. Skoff TH, Farley MM, Petit S, Craig AS, Schaffner W, Gershman K, et al. Increasing burden of invasive Group B Streptococcal disease in nonpregnant adults, 1990-2007. Clin Infect Dis. 2009. https://doi.org/10.1086/ 599369.

4. Gallagher PG, Watanakunakorn C. Group B streptococcal endocarditis: report of seven cases and review of the literature, 1962-1985. Rev Infect Dis. 1986. https://doi.org/10.1093/clinids/8.2.175.

5. Ballard MS, Schønheyder HC, Knudsen JD, Lyytikäinen O, Dryden M, Kennedy KJ, et al. The changing epidemiology of group B streptococcus bloodstream infection: a multi-national population-based assessment. Infect Dis. 2016. https://doi.org/10.3109/23744235.2015.1131330.

6. Schuchat A. Epidemiology of group B streptococcal disease in the United States: shifting paradigms. Clin Microbiol Rev. 1998. https://doi.org/10. 1128/CMR.11.3.497.

7. High KP, Edwards MS, Baker CJ. Group B streptococcal infections in elderly adults. Clin Infect Dis. 2005:41(6):839-47. https://doi.org/10.1086/432804.

8. Pimentel BAS, Martins CAS, Mendonça JC, Miranda PSD, Sanches GF, Mattos-Guaraldi AL, et al. Streptococcus agalactiae infection in cancer patients: a five-year study. Eur J Clin Microbiol Infect Dis. 2016. https://doi. org/10.1007/s10096-016-2617-9.

9. Sunkara B, Bheemreddy S, Lorber B, Lephart PR, Hayakawa K, Sobel JD, et al. Group B Streptococcus infections in non-pregnant adults: the role of immunosuppression. Int J Infect Dis. 2012. https://doi.org/10.1016/j. ijid.2011.11.008.

10. Georgieva RI, García López MV, Ruiz-Morales J, Martínez-Marcos FJ, Lomas JM, Plata A, et al. Streptococcus agalactiae left-sided infective endocarditis. Analysis of 27 cases from a multicentric cohort. J Infect. 2010. https://doi. org/10.1016/j.jinf.2010.04.005.

11. Rollán MJ, San Román JA, Vilacosta I, Sarriá C, López J, Acuña M, et al. Clinical profile of Streptococcus agalactiae native valve endocarditis. Am Heart J. 2003. https://doi.org/10.1016/S0002-8703(03)00444-7.

12. Hidalgo NF, Gharamti AA, Aznar ML, Almirante B, Yasmin M, Fortes CQ, et al. Beta-hemolytic streptococcal infective endocarditis: characteristics and outcomes from a large, multinational cohort. Open Forum Infect Dis. 2020. https://doi.org/10.1093/ofid/ofaa120.

13. Sambola A, Miro JM, Tornos MP, Almirante B, Moreno-Torrico A, Gurgui M, et al. Streptococcus agalactiae infective endocarditis: analysis of 30 cases and review of the literature, 1962-1998. Clin Infect Dis. 2002. https://doi. org/10.1086/340538.

14. Scully BE, Neu HC, Spriggs D. Streptococcus agalactiae (group B) endocarditis - a description of twelve cases and review of the literature. Infection. 1987. https://doi.org/10.1007/BF01646041.

15. Li JS, Sexton DJ, Mick N, Nettles R, Fowler VG, Ryan T, et al. Proposed modifications to the Duke criteria for the diagnosis of infective endocarditis. Clin Infect Dis. 2000. https://doi.org/10.1086/313753.

16. Blancas D, Santin M, Olmo M, Alcaide F, Carratala J, Gudiol F. Group B streptococcal disease in nonpregnant adults: incidence, clinical characteristics, and outcome. Eur J Clin Microbiol Infect Dis. 2004. https://doi. org/10.1007/s10096-003-1098-9.

17. Seo HS, Xiong YQ, Sullam PM. Role of the serine-rich surface glycoprotein Srr1 of Streptococcus agalactiae in the pathogenesis of infective endocarditis. PLoS ONE. 2013. https://doi.org/10.1371/journal.pone.0064204.

18. Oppegaard O, Mylvaganam H, Skrede S, Jordal S, Glambek M, Kittang BR. Clinical and molecular characteristics of infective $\beta$-hemolytic streptococcal endocarditis. Diagn Microbiol Infect Dis. 2017. https://doi.org/10. 1016/j.diagmicrobio.2017.06.015. 
19. Pringle SD, McCartney AC, Marshall DAS, Cobbe SM. Infective endocarditis caused by Streptococcus agalactiae. Int J Cardiol. 1989. https://doi.org/ 10.1016/0167-5273(89)90302-1.

20. Siciliano RF, Cais DP, Navarro RC, Varejão Strabelli TM. Acute Streptococcus agalactiae endocarditis: outcomes of early surgical treatment. Heart Lung J Acute Crit Care. 2010. https://doi.org/10.1016/j.hrtlng.2009.06.018.

21. Fischer B, Vojtila L, Rehm J. The 'fentanyl epidemic' in Canada—some cautionary observations focusing on opioid-related mortality. Prevent Med. 2018;107:109-13. https://doi.org/10.1016/j.ypmed.2017.11.001.

\section{Publisher's Note}

Springer Nature remains neutral with regard to jurisdictional claims in published maps and institutional affiliations.

- fast, convenient online submission

- thorough peer review by experienced researchers in your field

- rapid publication on acceptance

- support for research data, including large and complex data types

- gold Open Access which fosters wider collaboration and increased citations

- maximum visibility for your research: over $100 \mathrm{M}$ website views per year

At $\mathrm{BMC}$, research is always in progress.

Learn more biomedcentral.com/submissions 